\title{
CPG network to generate the swimming motion of the crawl stroke
}

\author{
Motomu NAKASHIMA*, Shogo FUJITA*, Takahiro MIYAZAWA** and Auke Jan IJSPEERT*** \\ ${ }^{*}$ Graduate School of Information Science and Engineering, Tokyo Institute of Technology \\ 2-12-2 Oookayama, Meguro-ku, Tokyo 152-8552, Japan \\ E-mail: motomu@sc.e.titech.ac.jp \\ ${ }^{* *}$ Graduate School of Science and Engineering, Tokyo Institute of Technology \\ ${ }^{* \star *}$ Ecole Polytechnique Federale de Lausanne (EPFL)
}

Received: 7 May 2016; Revised: 21 January 2017; Accepted: 25 April 2017

\begin{abstract}
The objective of this study was to propose a CPG network which can generate the swimming motion of the crawl stroke. First, the CPG network for legs performing a flutter kick was constructed by connecting the neural oscillators for the leg joints. The flutter kick motion was successfully generated by the proposed CPG network. The propulsion by the generated flutter kick motion was confirmed by the simulation of the swimming movement. Next, the CPG network for both the arms and legs were constructed, in which the neural oscillator for the arms initiated the trigger signal to start the prescribed stroke motion. By changing the intrinsic cycle of the neural oscillators for the legs, both six- and two-beat crawls could be realized. It was also found that a stable region with respect to the relationship between the intrinsic cycles of the neural oscillators for the arms and legs certainly existed for the six-beat crawl, although the intrinsic cycles of the arms were three times longer than those of the legs in this case. The propulsion by the generated swimming motion was confirmed by the simulation of the swimming movement both for the six- and two-beat crawls. Finally, the roll angle of the swimmer was fed back into the CPG network in order to restore the balance in the roll direction. Restoring the balance in the roll direction was successfully realized by the proposed feedback algorithm. The resultant motion showed a complicated behavior, such as skipping strokes.
\end{abstract}

Key words : Central pattern generator, Neural oscillator, Crawl stroke, Swimming, Sports engineering

\section{Introduction}

Rhythmic motions of animals, such as walking, running and swimming, are considered to be generated by the CPG (Central Pattern Generator), which exists in the spinal cord. The existence of the CPG was firstly suggested by the walking experiment of a decerebrated cat (Shik et al., 1966) and the study of lamprey (Grillner, 1985). The participation of the CPG in human walking was referred to the studies for the spinal-cord injured patients as well (Dimitrievic et al., 1998; Dietz et al., 2002).

In order to clarify the mechanism and characteristics of the CPG, neurophysiological approaches were conducted, as mentioned above. On the other hand, mathematical approaches by modeling the CPG as a nonlinear oscillating system were conducted (Wilson et al., 1972; Bay et al., 1987; Matsuoka, 1985; Matsuoka, 1987). The CPG model can reproduce the "entrainment" behavior of the CPG, which is a special characteristic to tune to a cyclic input signal. By utilizing this characteristic and constructing a CPG network with connecting multiple CPG models, it is possible to generate the complicated motion patterns where multiple joints move in a coordinated manner. In addition, by giving the sensor signals as feedback from the external environment to the CPG model, it is also possible to alter the output signals as well as the generated motion patterns into ones more adapted to the external environment.

The neural oscillator as a CPG model proposed by Matsuoka $(1985,1987)$ was frequently used, especially in the studies related to the relationship between the CPG and human walking. The simulation of bipedal walking in the sagittal plane was conducted by combining the neural oscillators and the musculoskeletal system (Taga et al., 1991; Taga et al., 
1994). In this simulation, it was confirmed that the adjustment of the gait was automatically performed during ascending and descending on a slope by incorporating the signals as feedback from the musculoskeletal system into the neural oscillators. This bipedal walking model was extended to both the frontal and sagittal planes by Miyakoshi et al. (1998). It was shown that stable stepping could be realized even with a perturbation force. Using the similar bipedal walking model by Taga's group, Hase and Yamazaki (1999) successfully generated the bipedal walking motion by solving the optimal combination of the connections and primal parameters of the neural oscillators by means of the Genetic Algorithm. Based on the results of these studies, neural oscillators were applied to control of the autonomous walking motion for the humanoid robots (Endo et al., 2005; Miura et al., 2012).

For both walking and the motions patterned to a high degree, the center of its execution is supposed to shift to a lower class in the central nervous system, such as the spinal cord and brainstem, while mastering the motion with repetition. Therefore, it was examined that the rhythm of the arms' cyclic motion has also been generated by the CPG (Zehr et al., 2003; Zehr et al., 2004a). Several studies, where the cyclic arm motions of humanoids were controlled by the neural oscillators, were reported as well (Williamson, 1998; Kotosaka et al., 2001; Yang et al., 2010). In addition, it was suggested that the coordinated motion of the arms and legs was possibly generated by the CPG (Zehr et al., 2004b).

As a good example of the coordinated motion of the arms and legs, swimming was focused on the present study. Humans move their arms and legs cyclically while swimming in a patterned motion, and a skilled swimmer seems to perform the swimming motion without thinking, although the swimming motion is very complicated especially for the arm stroke. These facts may suggest the possibility that the swimming motion is generated by the CPG. However, there are few studies related to human swimming from a neurophysiological viewpoint. If the subject species were not confined to human beings, the swimming motion of other animals were in the scope of the CPG study from the beginning. The swimming motion of lamprey was investigated by Grillner (1985), as mentioned above. For salamanders, Ijspeert et al. (2007) demonstrated a neurophysiological model which could explain the automatic transition from walking to swimming by using phase oscillators. However, for human swimming, only Wannier et al. (2001) suggested the relationship between the CPG and the motion pattern of the arms and legs in crawl swimming by means of a neurophysiological experiment, although the detailed mechanism for it was not described.

The objective of this study was to propose a CPG network which can generate the swimming motion of the crawl stroke. The crawl stroke is the most popular and fastest among all strokes. An interesting feature of the crawl stroke is that the six-beat kick style is normally used. In the six-beat kick style, two strokes by both arms and six kicks by both legs are performed in one stroke cycle. It means that the cycle of the arms is three-times longer than that of the legs. It has not been reported yet that such different cycles between the arms and legs can be realized successfully by a CPG network. Such a CPG network has been proposed in the present study. The present study contributes to the understanding of how a human generates the swimming motion, as well as further investigation of the CPG network. In addition, the present study also contributes to the control method of a humanoid robot since a swimming humanoid robot was recently developed (Chung and Nakashima, 2013a, 2013b).

This paper is organized as follows: Generation of the flutter kick motions for the legs are described in $\S 2$. Coordination of the arms and legs is discussed in $\S 3$. The roll angle feedback to restore the balance is demonstrated in $\S$ 4. The conclusions and suggestions obtained in this study are summarized in $\S 5$.

\section{Generation of flutter kick motion for legs}

\subsection{Basic CPG mechanism}

As the mathematical model of the CPG in the present study, the neural oscillator proposed by Matsuoka $(1985,1987)$ was used. The neural oscillator is a model of the excitation/inhibition mechanism between the neurons, which is seen in the neural circuit of the CPG. The basic structure is a nonlinear oscillator in which two neuron models having the adaptation (self-inhibition, fatigue) effect are paired as the mutual inhibition network. When a constant input signal is put into one neuron, the output signal approaches the constant value once, and then starts decaying. The neuron model is represented by a set of the first order differential equations as follows:

$$
\tau \dot{x}^{i}+x^{i}=-\sum_{j=1}^{n} w^{i j} y^{j}-b v^{i}+s^{i}+\sum k^{i} g^{i}
$$




$$
\begin{aligned}
& T \dot{v}^{i}+v^{i}=y^{i} \\
& y^{i}=\left[x^{i}\right]^{+}=\max \left(x^{i}, 0\right)
\end{aligned}
$$

where $x^{i}$ is the membrane potential of the neuron body and $v^{i}$ is an internal variable. The symbols $\tau$ and $T$ are the time constants, $b$ is the fatigue coefficient, $w^{i j}$ is the weighting coefficient for the connection between the neurons, $s^{i}$ is a constant input. In addition, $k^{i}$ is the feedback gain and $g^{i}$ is the feedback signal. Finally, $y^{i}$ is the output signal from the neuron. These differential equations are solved by time integration (Runge-Kutta method) for the numerical simulations. A mutual inhibition network consisting of two neuron models (a neural oscillator) is shown in Fig. 1.

\subsection{CPG network to generate a flutter kick motion}

For the flutter kick of the crawl stroke, the flexion/extension motions of the hip, knee and ankle joints were considered. The actual joint angles of the right leg for a competitive swimmer are shown in Fig. 2. These were measured from the video analysis and used for the simulation study by Nakashima et al. (2007). From this figure, the phase differences between the hip, knee and ankle joints were found to have a simple relationship. That is, the knee joint lags behind the hip joint for one-sixth of the kick cycle, and the ankle joint lags behind the knee joint for one-sixth of the kick cycle as well. In addition, the right and left legs have an anti-phase relationship, of course. Based on these facts, the CPG network for the flutter kick was proposed as shown in Fig. 3. Each joint has two mutually inhibited neurons for extension and

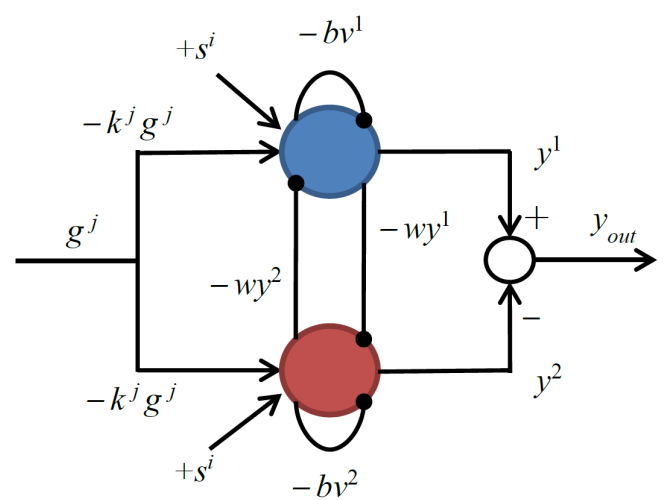

(a) Detailed notation

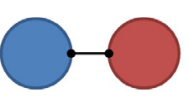

(b) Simple notation

Fig. 1 A neural oscillator as a mutual inhibition network consisting of two neuron models.

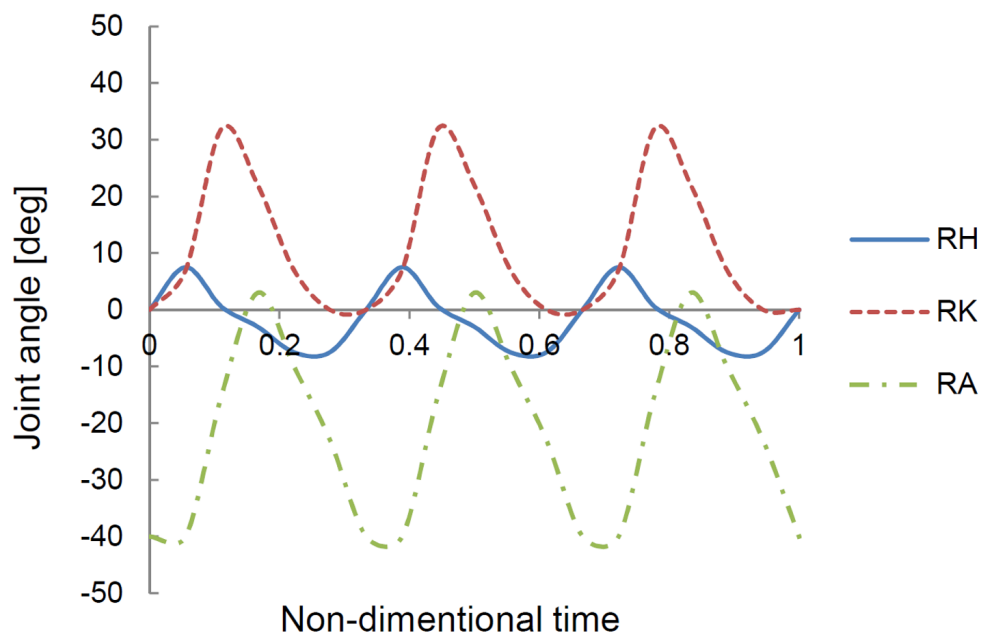

Fig. 2 Actual joint angles of the right leg of a competitive swimmer measured in Nakashima et al. (2007). RH: Hip. RK: Knee. RA: Ankle. 
flexion of the joint, which are denoted by "_e" and "_f" in the figure, respectively. The output signal of the neural oscillator for each joint was calculated as the difference between the output signals from the two neurons, that is,

$$
y=y_{e}-y_{f}
$$

A pair of three neurons RH_f, RK_e and RA_f was connected with a cyclic inhibition, and another pair of RH_e, RK_f and RA_e was also connected in the same manner. These connections enabled the one-sixth phase lags. In addition, RH_e and LH_e, as well as RH_f and LH_f, were connected with mutual inhibition. These connections enabled the anti-phase relationship between the right and left legs. An example of the output signals from this CPG network is shown in Fig. 4. The parameters in this example were given as follows: $\tau_{1}=0.098 \mathrm{~s}, T_{1}=3 \tau_{1}, b_{1}=2.50, s_{1}=5.0$ (the subscript " 1 " represents "common for all connections among the neurons of the legs."), $w_{m}=1.5$ (weighting coefficient for the mutual inhibiting connections, for example, the connection between $\mathrm{LH}_{-}$e and $\mathrm{LH} \_$), and $w_{c}=1.2$ (weighting coefficient for the cyclic inhibiting connections, for example, the connection between LH_e and LK_f). It was confirmed that the three joints for each leg had a one-sixth phase lag relationship, and that the right and left legs had a successful anti-phase relationship. The cycle of the flutter kick was $0.66 \mathrm{~s}$. Note that the parameters for this example were determined by trial and error, so that the output curves became similar to those of Fig. 2.

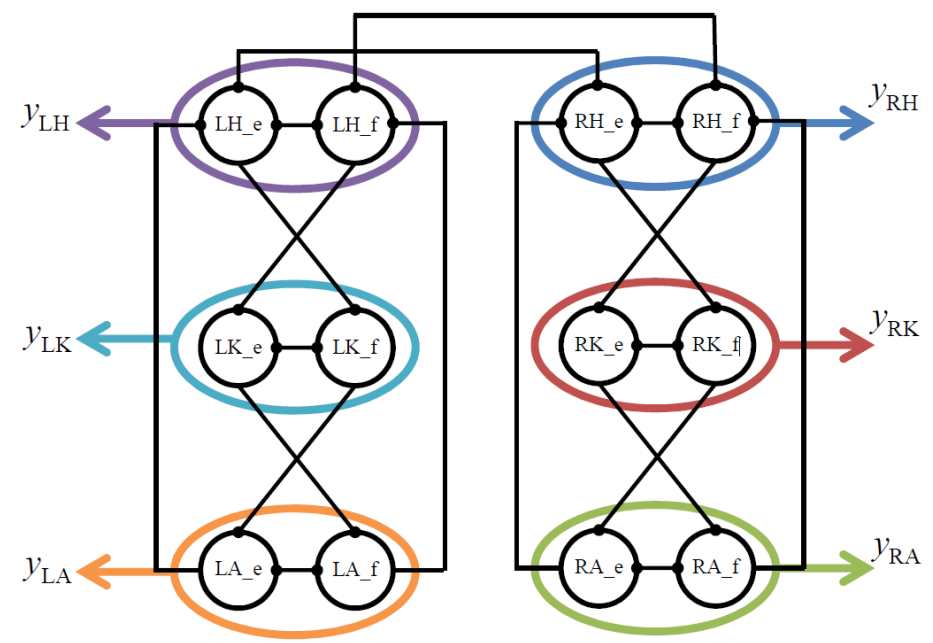

Fig. 3 CPG network for the legs to realize the flutter kick. R: Right. L: Left. H,K,A: Hip, Knee and Ankle, respectively.

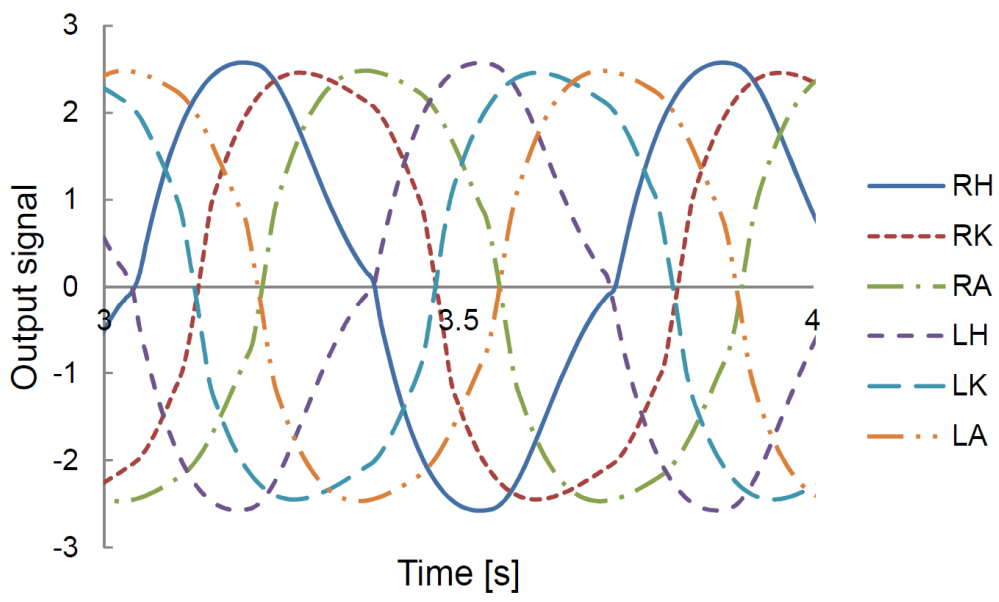

Fig. 4 Output signals from the proposed CPG network. 


\subsection{Simulation of swimming movement}

The output signals from the neural oscillator were converted into the joint angles by using a simple linear transformation (magnification and offset) as follows:

$$
\begin{aligned}
& \theta_{R H}=\theta_{R H 0}+k_{R H} y_{R H} \\
& \theta_{R K}=\theta_{R K 0}+k_{R K} y_{R K} \\
& \theta_{R A}=\theta_{R A 0}+k_{R A} y_{R A}
\end{aligned}
$$

The parameters $\theta_{R H 0}, k_{R H}, \theta_{R K 0}, k_{R K}, \theta_{R A 0}$ and $k_{R A}$ are the coefficients for the offset and magnification for the right hip, knee and ankle joints, respectively. The similar equations holds for the left leg as well. The parameters were determined so that the resultant joint angles became sufficiently similar to the actual values shown in Fig. 2 . The joint angles with the determined parameters are shown in Fig. 5. It was confirmed that the amplitude and offset obtained from the CPG network were sufficiently consistent with the actual values shown in Fig. 2 although the curve shapes were somewhat different from those of Fig. 2.

A simulation of swimming movement with the obtained joint angles was conducted. The simulation model "SWUM" was used for the simulation. SWUM was developed for the mechanical analysis of human swimming (Nakashima et al., 2007). In SWUM, the swimmer's body is represented as a series of 21 truncated elliptic cones, as shown in Fig. 6 . The fluid forces acting on the swimmer are assumed to be computable from the local motion of the swimmer's body. By inputting the body geometry and joint motion, the equations of motion of the swimmer as one rigid body are solved by means of the time-marching method. As a result, the user can obtain the fluid forces acting on the swimmer and the resultant swimming movement, such as swimming speed. Various studies by means of SWUM including its validations have already been conducted (Nakashima, 2007; Nakashima, 2010).

As the simulation conditions, 10 seconds were simulated with the time interval of $1 / 500 \mathrm{~s}$. The joint angles shown in Fig. 5 were put into the simulation for the legs, while the arms were fixed in the stretched position. For other simulation

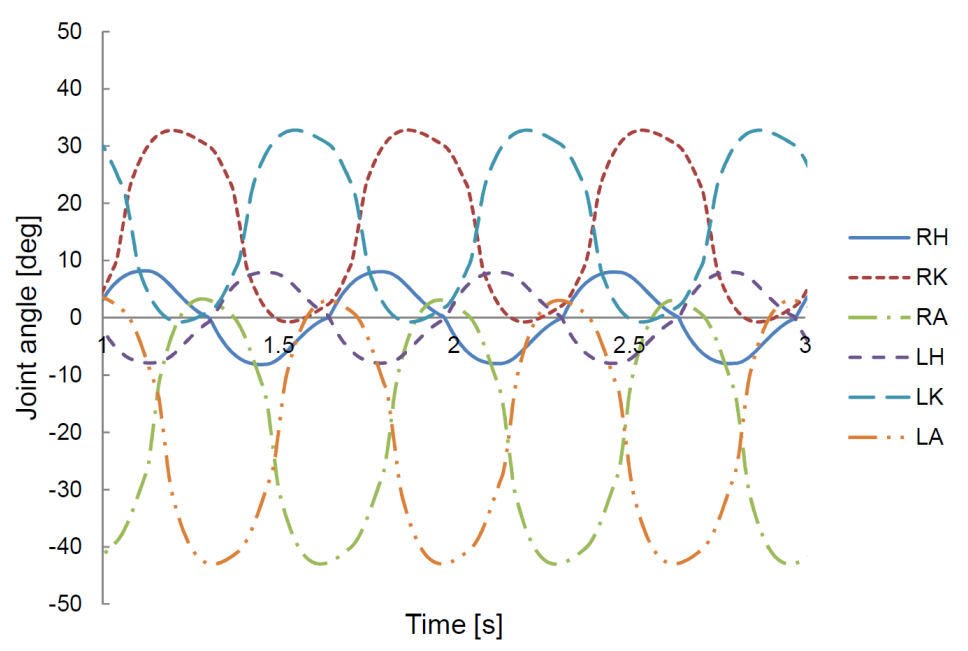

Fig. 5 Joint angles generated from the output signals in the proposed CPG network.

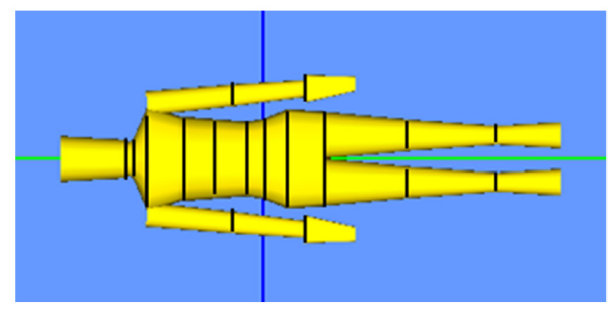

Fig. 6 Representation of a human body in SWUM. 
conditions, such as the body geometry of the swimmer as well as the coefficients in the fluid force model, the standard model parameters, which were used in the previous studies (Nakashima et al., 2007; Nakashima, 2007), were used in the present simulation as well.

The animation images of the simulated swimming movement are shown in Fig. 7. The pale blue horizontal line close to the swimmer represents the water surface. The red lines emerging from the feet represent the directions and magnitudes of the fluid forces. It was confirmed that the swimmer successfully propels by the flutter kick. The resultant swimming velocity was found to be $0.75 \mathrm{~m} / \mathrm{s}$.

\section{Coordination of arms and legs}

\subsection{Proposed CPG network}

The stroke motion by the arms is much more complicated than the flutter kick by the legs. Therefore, only the starting timing of each stroke motion by each arm was controlled by the CPG network in the present study. The proposed CPG network is shown in Fig. 8. The two upper neural oscillators are for the arms. Since they are connected to each other, they can generate the rhythmic outputs by themselves. In addition, the neural oscillators for the arms were connected to those for the hip joints, as shown in Fig. 8.

The output signals from the proposed CPG network for the six-beat crawl are shown in Fig. 9. In this example, the parameters for the legs were the same as the ones shown in Fig. 4. For the CPG network for the arms, the following parameters were given: $\tau_{2}=0.147 \mathrm{~s}, T_{2}=3 \tau_{2}, b_{2}=2.50, s_{2}=19.0, w_{2}=1.5$. Note that the subscript " 2 " represents "common for all connections among the neurons of the arms." This parameter set gave the cycle of $1.98 \mathrm{~s}$, that is, three times of that of the legs. The weighting coefficient for the mutual inhibiting connection between the arms and legs was given as $w_{3}=0.30$, which was determined through trial and error. From the figure, it was found that the output signals of

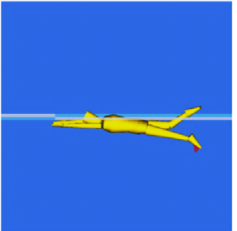

$t=8.50 \mathrm{~s}$



$t=8.86 \mathrm{~s}$

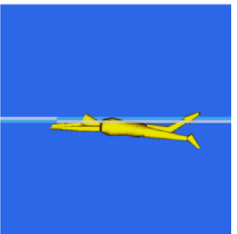

$t=8.56 \mathrm{~s}$

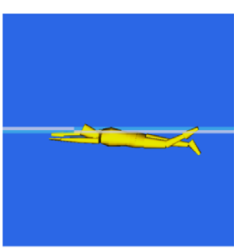

$t=8.92 \mathrm{~s}$

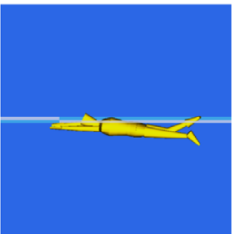

$t=8.62 \mathrm{~s}$

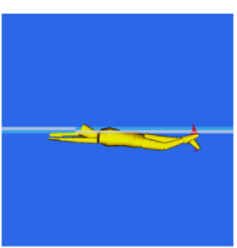

$t=8.98 \mathrm{~s}$

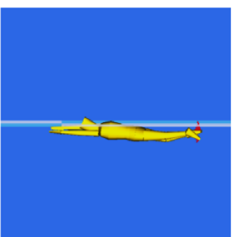

$t=8.68 \mathrm{~s}$

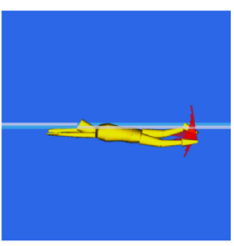

$t=9.04 \mathrm{~s}$



$t=8.74 \mathrm{~s}$

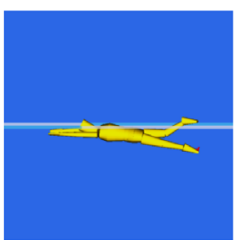

$t=9.10 \mathrm{~s}$

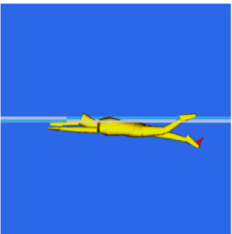

$t=8.80 \mathrm{~s}$

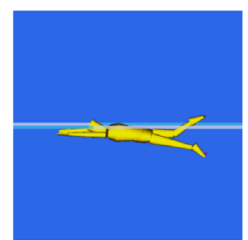

$t=9.16 \mathrm{~s}$

Fig. 7 Animation images of the simulated swimming movement.

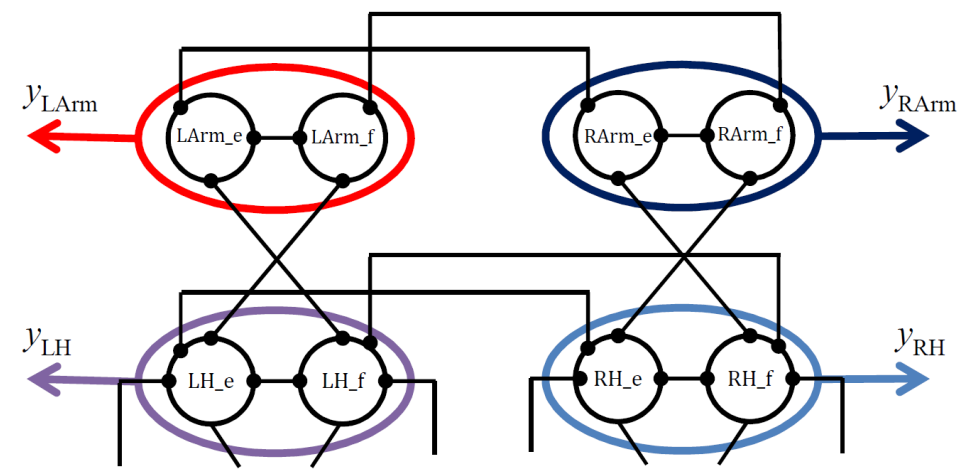

Fig. 8 CPG network for the coordination of arms and legs. 


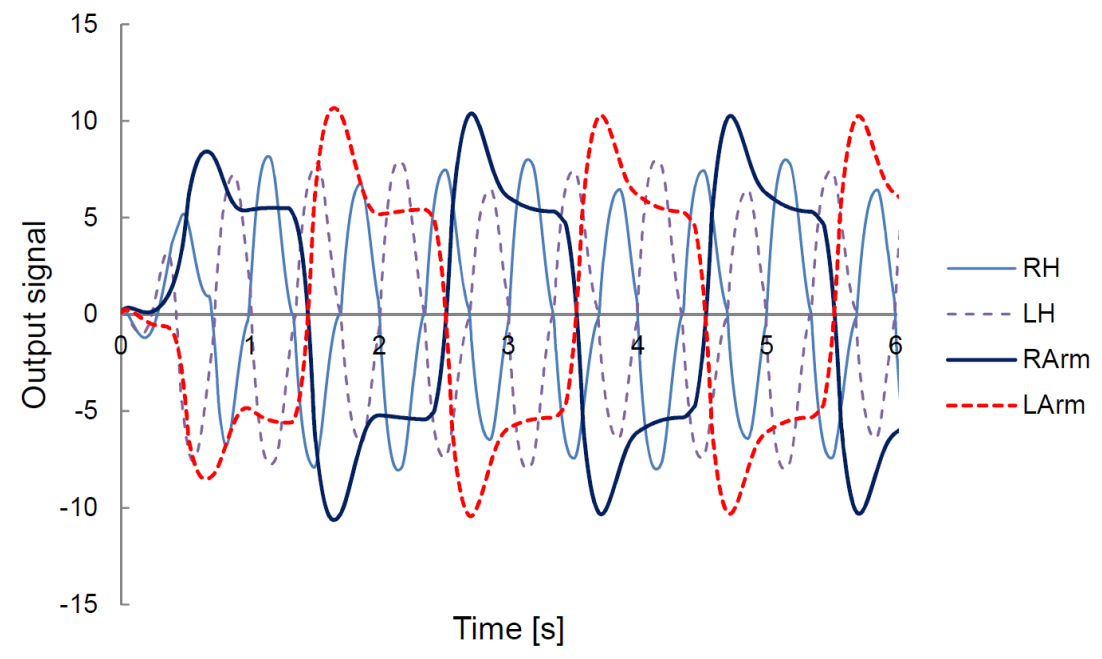

Fig. 9 Output signals from the proposed CPG network for the six-beat crawl.

the hip joints were slightly deformed, that is, the amplitude was not constant but had slight fluctuation during one stroke cycle. The fluctuation was due to the mutual inhibiting connection between the arms and legs. Although this slight deformation was observed, it was confirmed that the stable oscillation was generated by the proposed CPG network.

\subsection{Characteristics of entrainment behavior}

The stable oscillation observed in the previous section was realized due to the entrainment behavior of the CPG network. Therefore, the detailed characteristics of the entrainment were investigated by changing the intrinsic cycle of the CPG network for the legs. Holding the relationship of $T_{1}=3 \tau_{1}$, the time constant for the legs $\tau_{1}$ was changed. As a result, the stable oscillation of the six-beat crawl was obtained within the range of $\tau_{1}=0.088 \sim 0.127 \mathrm{~s}$. The width of this range shows the performance of the entrainment and therefore the robustness against disturbances of the CPG network as well.

Interestingly, a two-beat crawl motion (two kicks in one stroke cycle) was obtained when the time constant $\tau_{1}$ was increased further. The two-beat crawl motion was obtained in the range of $\tau_{1}=0.225 \sim 0.627 \mathrm{~s}$, which was much broader than that for the six-beat crawl. In addition, the phase relationship between the arms and legs changed according to the change in $\tau_{1}$ within the range of $\tau_{1}=0.225 \sim 0.294 \mathrm{~s}$. The output signals in the case of $\tau_{1}=0.225,0.260$ and $0.294 \mathrm{~s}$ are shown in Figs. 10, 11 and 12, respectively. It was confirmed that the phase relationship between the arms and legs changed according to the change in $\tau_{1}$. This characteristic is useful to control the phase relationship when these signals are utilized for the swimming motion.

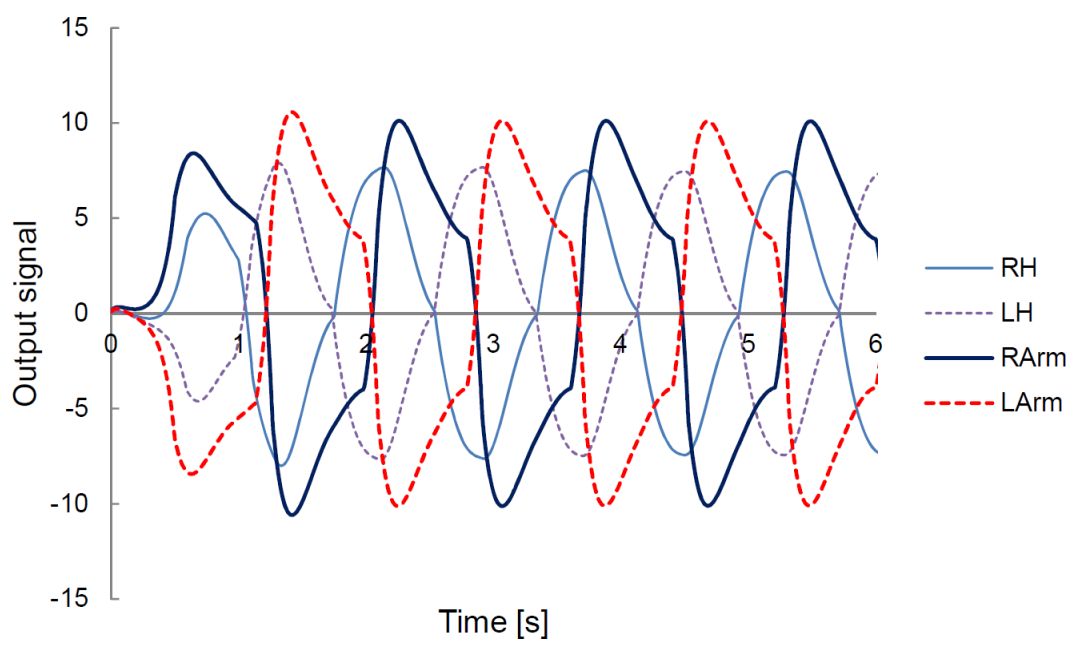

Fig. 10 Output signals from the proposed CPG network for the two-beat crawl $\left(\tau_{1}=0.225\right)$. 


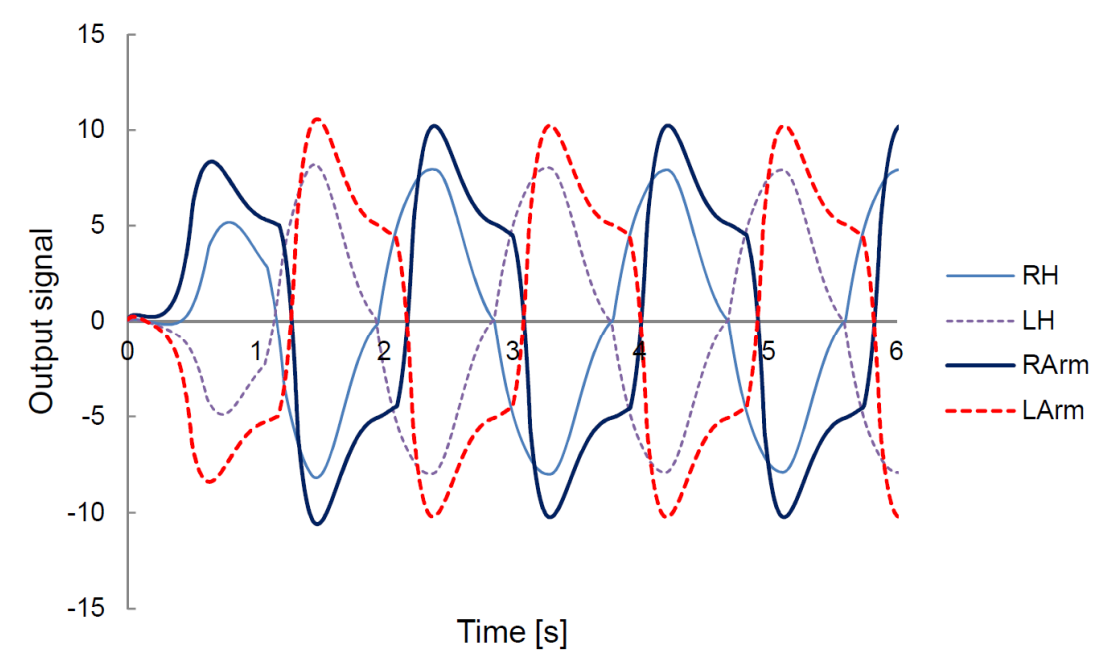

Fig. 11 Output signals from the proposed CPG network for the two-beat crawl $\left(\tau_{1}=0.260\right)$.

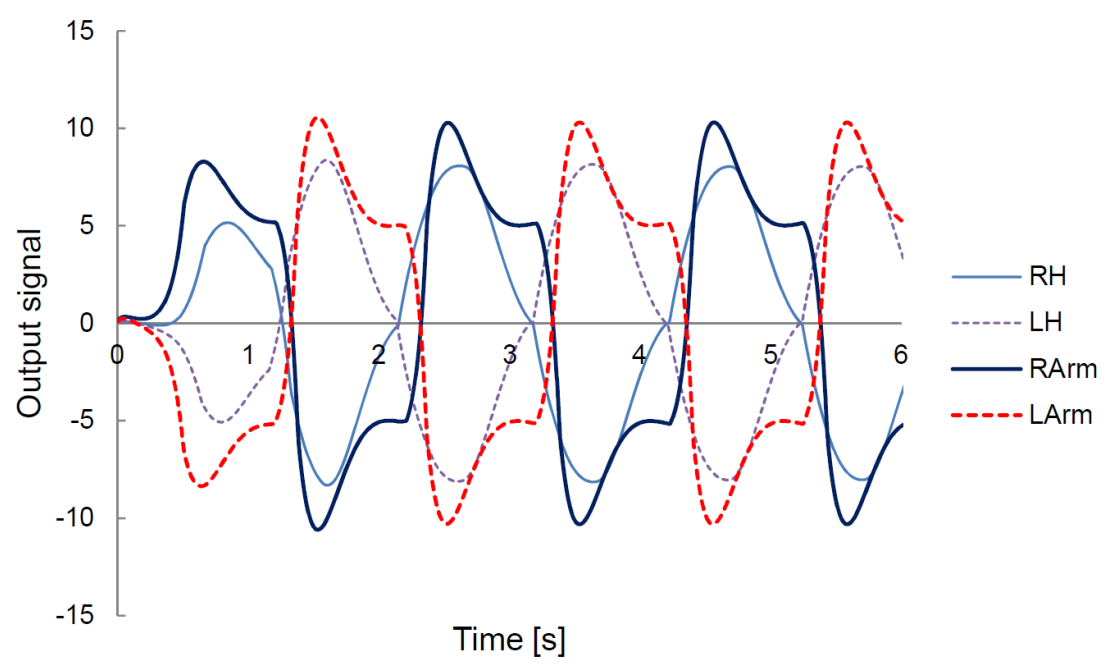

Fig. 12 Output signals from the proposed CPG network for the two-beat crawl $\left(\tau_{1}=0.294\right)$.

The detailed relationship between $\tau_{1}$ and the resultant output from the CPG network was investigated by changing $\tau_{1}$ to the further broader range. In order to distinguish the stable and unstable oscillations systematically, a deviation of the resultant cycle length of the neural oscillator for the arm was utilized. For example, the resultant cycle lengths for half cycles of the arm neural oscillator were plotted in Fig. 13. The case of stable oscillation is shown in Fig. 13(a) ( $\tau_{1}$ $=0.098 \mathrm{~s})$ while that of the unstable oscillation is shown in Fig. 13(b) $\left(\tau_{1}=0.137 \mathrm{~s}\right)$. These values were calculated by recording the timings when the output signal from the right arm neural oscillator became zero and obtained the differences between the adjacent two timings. Therefore, these values were obtained for each half cycle, as shown in the abscissa of Fig. 13. It was confirmed that the cycle length became constant in the stable oscillation as shown in Fig. 13(a) while it fluctuated in the unstable case as shown in Fig. 13(b). From this result, it was found that the stability of the oscillation could be evaluated by the magnitude of the fluctuation. If the oscillation is stable, the fluctuation becomes sufficiently small. Therefore, the differences of the cycle lengths between the last two adjacent cycle numbers ( 3.5 and 4 in the actual calculation) were taken and plotted in Fig. 14. The abscissa is the time constant $\tau_{1}$ and the ordinate is the difference of the cycle lengths. The narrow stable (small differences) range of $\tau_{1}=0.088 \sim 0.127$ corresponds to the six-beat crawl. The broader range of $\tau_{1}=0.225 \sim 0.627$ corresponds to the two-beat crawl. From this figure, it was found that the stable range inducing the entrainment exists for the six-beat crawl, which has three times the different intrinsic cycles than the arms and legs, although the range is narrower than that of the two-beat crawl, which almost has the same intrinsic cycles 


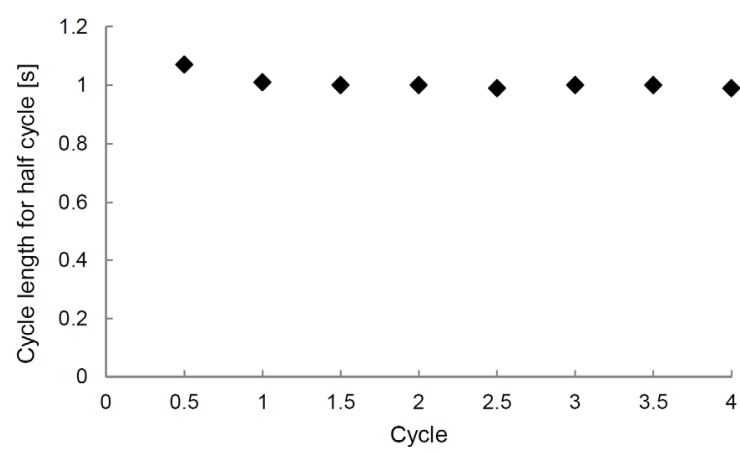

(a) stable oscillation $\left(\tau_{1}=0.098 \mathrm{~s}\right)$

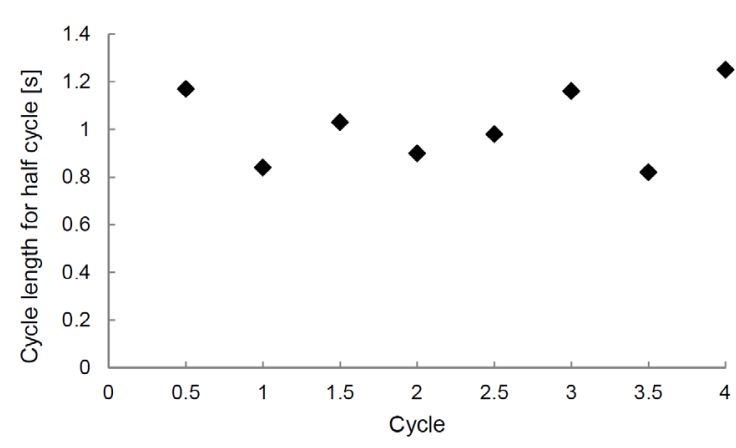

(b) unstable oscillation $\left(\tau_{1}=0.137 \mathrm{~s}\right)$

Fig. 13 Cycle lengths for half cycles of the arm oscillator.

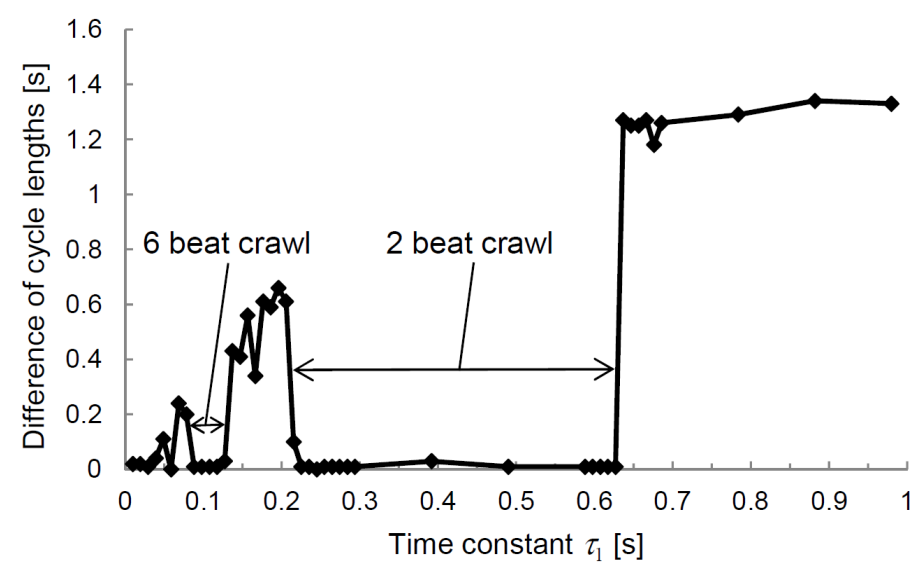

Fig. 14 Relationship between time constant $\tau_{1}$ and differences of the cycle lengths between the last two adjacent half cycles.



Fig. 15 Relationship between time constant $\tau_{1}$ and the cycle lengths for the right arm.

for the arms and legs. Note that there was a possibility that the difference of cycle lengths happened to become zero even in the case of unstable oscillation, since it was calculated just as a difference between the cycle lengths of 3.5-th and 4.0th cycle. However, there was no possibility that the difference happened to take large value in the case of stable oscillation. Just to make sure, whether the cycle length became constant value or not (stable or not) was manually checked for the ranges of the six- and two-beat crawl, which were shown in Fig. 14. As a result, the stable oscillation was certainly confirmed for these ranges. The resultant cycle lengths for the right arm in the function of $\tau_{1}$ are shown in Fig. 15. In 
this figure, the intrinsic cycle length of the arm was a constant value of $1.98 \mathrm{~s}$. However, the arms' neural oscillators were entrained by the legs' neural oscillators. In particular, it was found that the resultant cycle length changed linearly in the two ranges of stable oscillation, that is, $\tau_{1}=0.088 \sim 0.127 \mathrm{~s}$ for the six-beat crawl and $\tau_{1}=0.225 \sim 0.627 \mathrm{~s}$ for the twobeat crawl.

\subsection{Simulation of the swimming movement}

The simulation of the swimming movement for the crawl stroke was conducted. With respect to the arm motion, the output signals from the CPG network were only used as a "trigger" to specify the starting time of the prescribed stroke motion. The prescribed stroke motion is shown in Fig. 16. The symbol $t$ ' is the elapsed time from the starting time of the stroke. The default position is the one at $t^{\prime}=0.0 \mathrm{~s}$, that is, the arm stretched in the forward position. If the next output signal does not come, the arm returns to this position after the stroke.

With respect to the starting trigger, it was found that the output signals for the arms slightly exceeded 10 at their peaks, as shown in Fig. 9. This timing was used for the trigger. When the output signal exceeded 10 from the value below it, the prescribed stroke motion was initiated. Therefore, if the output signal did not exceed 10 at the peak, the stroke motion of the corresponding arm was not started for that cycle.

With respect to the leg motion, the parameters for the CPG network of Fig. 9 in the previous sections were used for the six-beat crawl. The relationship between the output signals from the CPG network and the joint angles was also the same as that used in the simulation of the flutter kick, as shown in Eq. (5) (7). For the two-beat crawl, on the other hand, a different strategy was taken to generate the joint motions. Since the number of kicks in the two-beat crawl is less than those in the six-beat crawl, each kick has to generate a larger fluid force effectively. In the crawl stroke, the flutter kick has an important role to raise the lower body upward (Nakashima, 2007). If the fluid force by the flutter kick is insufficient, the lower body sinks, and it prevents the propulsion. Indeed, by a preliminary simulation, it was found that the modeled human did not swim well using the joint angles calculated from Eqs. (5), (6) and (7) since the lower body sunk. Therefore, the following equations were applied based on the observation of an actual swimmer performing the two-beat crawl.

$$
\begin{aligned}
& \theta_{R H}[\mathrm{deg}]=\left\{\begin{array}{cc}
12+0.1 \times\left(y_{R H}-1.2\right) & \left(y_{R H} \geq 1.2\right) \\
10 \times y_{R H} & \left(\left|y_{R H}\right|<1.2\right) \\
-12+0.1 \times\left(y_{R H}+1.2\right) & \left(y_{R H} \leq-1.2\right)
\end{array}\right. \\
& \theta_{R K}[\mathrm{deg}]=\left\{\begin{array}{cc}
0.12 \times\left(y_{R K}-1.2\right)^{3} & \left(y_{R K} \geq 1.2\right) \\
0 & \left(y_{R K}<1.2\right)
\end{array}\right. \\
& \theta_{R A}[\mathrm{deg}]=\theta_{R A 0}+k_{R A} y_{R A}
\end{aligned}
$$

The same equations were applied to the left leg as well. The joint angles of the legs for the two-beat crawl are shown in Fig. 17. By the above-mentioned transformation, it was found that the hip joint angle was close to a square wave, which meant that the transition between the flexed and extended positions was abrupt. It was also found that the knee joint angle was close to a triangle wave.

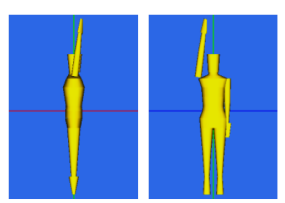

$t^{\prime}=0.0 \mathrm{~s}(1.98 \mathrm{~s})$

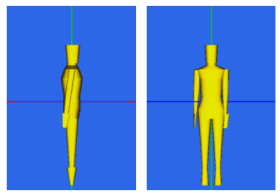

$t^{\prime}=0.99 \mathrm{~s}$



$t^{\prime}=0.33 \mathrm{~s}$

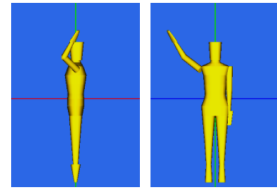

$t^{\prime}=1.32 \mathrm{~s}$



$t^{\prime}=0.66 \mathrm{~s}$

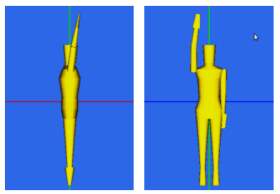

$t^{\prime}=1.65 \mathrm{~s}$

Fig. 16 Prescribed stroke motion for arms. 


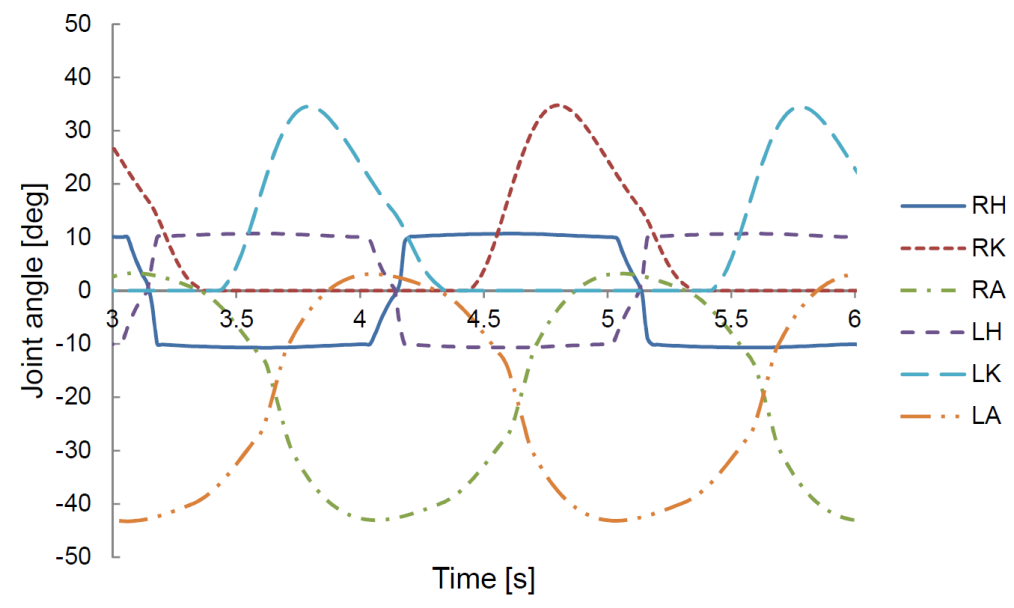

Fig. 17 Joint angles of the legs for two-beat crawl.

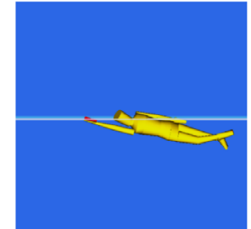

$t=17.60 \mathrm{~s}$

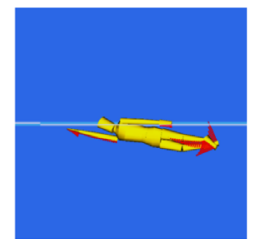

$t=18.68 \mathrm{~s}$

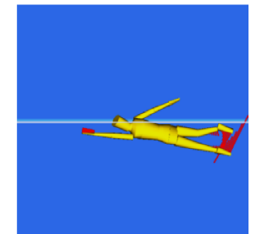

$t=17.78 \mathrm{~s}$

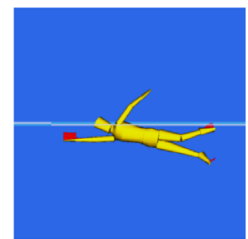

$t=18.86 \mathrm{~s}$



$t=17.96 \mathrm{~s}$

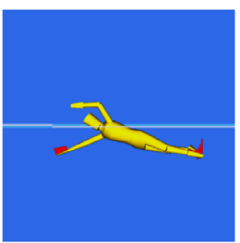

$t=19.04 \mathrm{~s}$

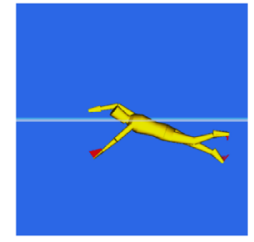

$t=18.14 \mathrm{~s}$



$t=19.22 \mathrm{~s}$

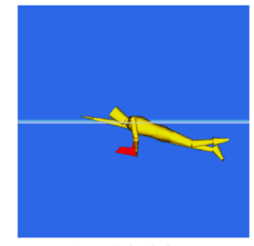

$t=18.32 \mathrm{~s}$

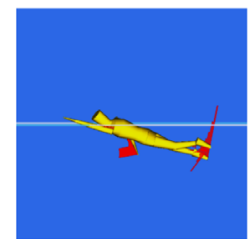

$t=19.40 \mathrm{~s}$

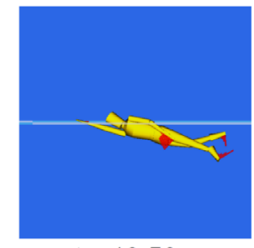

$t=18.50 \mathrm{~s}$

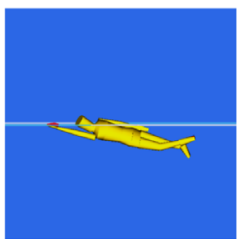

$t=19.58 \mathrm{~s}$

Fig. 18 Animation images of the simulated swimming movement (six-beat crawl).



$t=17.26 \mathrm{~s}$

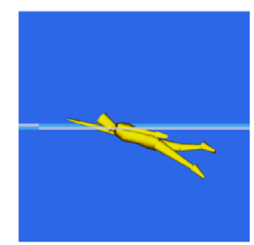

$t=18.34 \mathrm{~s}$

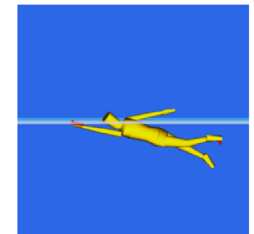

$t=17.44 \mathrm{~s}$

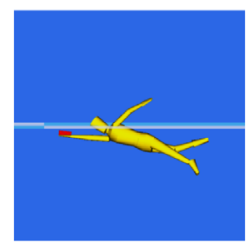

$t=18.52 \mathrm{~s}$

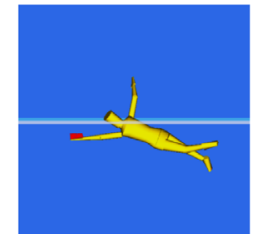

$t=17.62 \mathrm{~s}$

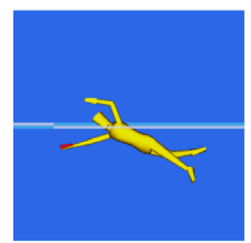

$t=18.70 \mathrm{~s}$

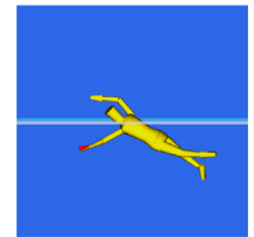

$t=17.80 \mathrm{~s}$



$t=18.88 \mathrm{~s}$

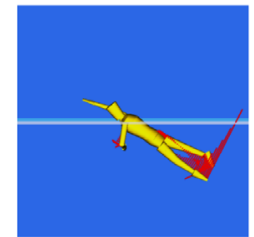

$t=17.98 \mathrm{~s}$

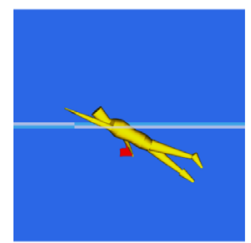

$t=19.06 \mathrm{~s}$

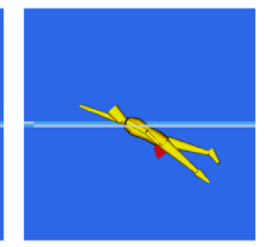

$t=18.16 \mathrm{~s}$

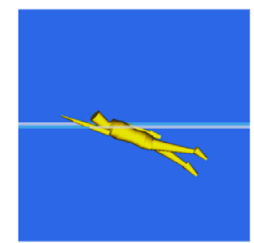

$t=19.24 \mathrm{~s}$

Fig. 19 Animation images of the simulated swimming movement (two-beat crawl).

By using the joint angles described above, simulations of the swimming movements were conducted for the six- and two-beat crawls. The animation images of the simulated swimming movements for the six- and two-beat crawls are shown in Figs. 18 and 19, respectively. It was confirmed that both the six- and two-beat crawls were successfully realized by the proposed CPG network as well as the joint angle transformation. From the comparison between Figs. 18 and 19 , 
it was found that the lower half of the body became lower (sunken) in the two-beat crawl. The reason for this is that the fluid force generated by the flutter kick was still not sufficient in the two-beat crawl. The averaged swimming velocities of the six- and two-beat crawls were obtained as $1.20 \mathrm{~m} / \mathrm{s}$ and $0.97 \mathrm{~m} / \mathrm{s}$, respectively. The reason for the lower swimming velocity in the two-beat crawl was that the lower half of the body became lower, as mentioned above.

\section{Roll angle feedback to restore the balance}

\subsection{Proposed CPG network}

In the proposed CPG network, the roll signals were fed back into the neurons for the arms as follows:

For LArm_e and RArm_f :

$$
\begin{aligned}
& \tau_{2} \dot{x}^{i}+x^{i}=-\sum_{j=1}^{n} w^{i j} y^{j}-b_{2} v^{i}+s_{2}-k_{f b}[\text { Roll }]^{+} \\
& T_{2} \dot{v}^{i}+v^{i}=y^{i} \\
& y^{i}=\left[x^{i}\right]^{+}=\max \left(x^{i}, 0\right) \\
& {\left[\text { Roll }^{+}=\left\{\begin{array}{cc}
\theta_{\text {roll }}-\theta_{\text {th }} & \left(\theta_{\text {roll }} \geq \theta_{\text {th }}\right) \\
0 & \left(\theta_{\text {roll }}<\theta_{\text {th }}\right)
\end{array}\right.\right.}
\end{aligned}
$$

For RArm_e and LArm_f :

$$
\begin{aligned}
& \tau_{2} \dot{x}^{i}+x^{i}=-\sum_{j=1}^{n} w^{i j} y^{j}-b_{2} v^{i}+s_{2}-k_{f b}[\text { Roll }]^{-} \\
& T_{2} \dot{v}^{i}+v^{i}=y^{i} \\
& y^{i}=\left[x^{i}\right]^{+}=\max \left(x^{i}, 0\right) \\
& {[\text { Roll }]^{-}=\left\{\begin{array}{cc}
0 & \left(\theta_{\text {roll }}>-\theta_{\text {th }}\right) \\
-\theta_{\text {roll }}-\theta_{\text {th }} & \left(\theta_{\text {roll }} \leq-\theta_{\text {th }}\right)
\end{array}\right.}
\end{aligned}
$$

The symbols $\theta_{\text {roll }}$ and $\theta_{t h}$ represent the roll angle of the swimmer's body and the threshold angle of the roll, respectively. The CPG network for this feedback algorithm is shown in Fig. 20. This feedback algorithm was devised

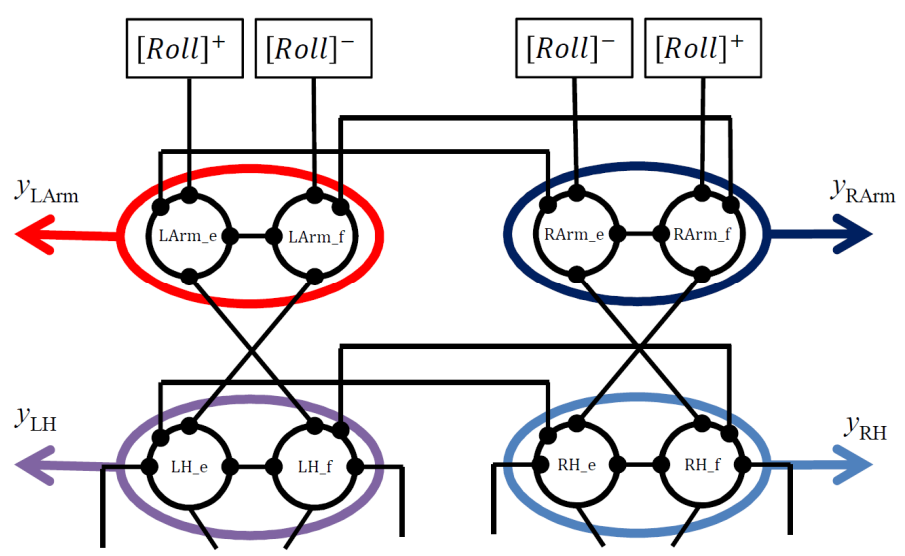

Fig. 20 Proposed CPG network for roll angle feedback. 
utilizing the feature that a human body is statically stable in the roll direction. This stability comes from the shape of a human body. Generally, in the swimming position, four limbs are horizontally aligned, and the trunk has horizontally wide elliptic shape. For such shape, more restoring moment by buoyancy acts on the body than inclining moment by gravity. Therefore, when some disturbance acts on the human body during swimming, it is effective to delay (or stop) the arm stroke until the roll angle becomes small. For example, let us consider the case in which the roll angle $\theta_{\text {roll }}$ becomes larger than the threshold angle $\theta_{t h}$. The large $\theta_{\text {roll }}$ means the right shoulder sinks deeply. In this case, the neurons LArm_e and RArm_f were inhibited by the feedback term $k_{f b}[R o l l]^{+}$. By this inhibition, the output signals from LArm_e were difficult to make large, and those from RArm_f were difficult to make small. As a result, the timing of exceeding 10 for the left arm was delayed, or did not exceed 10 at all, since LArm_e is in charge of the positive value of the output signal from the neural oscillator for the left arm. When the right shoulder sinks deeper, more time is necessary to recover. The proposed feedback algorithm provides this extra time to recover. In addition, this algorithm also provides skipping the left arm stroke in some case. This skipping also contributes to reduce $\theta_{\text {roll }}$ since the left arm stroke has an effect of increasing $\theta_{\text {roll }}$.

\subsection{Simulation of the swimming movement}

The simulation of swimming movement subjected to a disturbance was conducted. The input parameters identical to those of the six-beat crawl, which are shown in $\S 3.3$, were given for the simulation in this section as well. As the disturbance, the angular momentum of $0.30 \mathrm{Nm} / \mathrm{s}$ in the positive roll direction was given for the period of $t=12.50 \sim$ $12.60 \mathrm{~s}$.

First, the simulation without feedback was conducted. The result of the roll angle is shown in Fig. 21. From this figure, it was found that the roll angle did not decrease $(t=13.5 \mathrm{~s})$, further increased due to the next left arm stroke (15.0 $\mathrm{s})$, and finally the swimmer totally lost balance and turned over $(20.0 \mathrm{~s})$. Therefore, it was confirmed that the swimmer could not keep swimming without the feedback.

Next, the simulation with the roll angle feedback was conducted. The parameters for the feedback were given as $\theta_{t h}$ $=50^{\circ}$ and $k_{f b}=25.0$. These values were determined through trial and error, so that the feedback worked well. The results of the output signals and roll angle with feedback are shown in Fig. 22. The animation images of the simulated swimming



Fig. 21 Roll angle in swimming subjected to a disturbance (without feedback). The green vertical line represents the period of the disturbance $(t=12.50 \sim 12.60 \mathrm{~s})$.

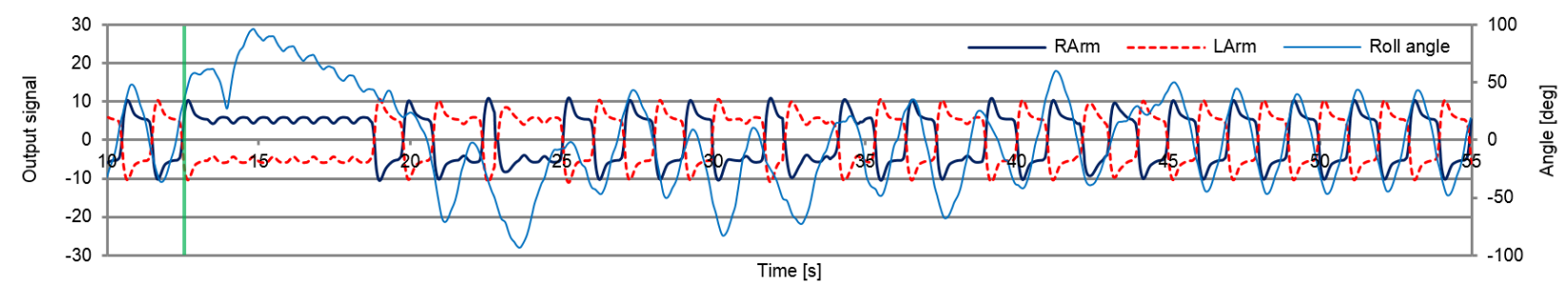

Fig. 22 Output signals and roll angle (with feedback). The green vertical line represents the period of the disturbance $(t=12.50 \sim 12.60 \mathrm{~s})$. 


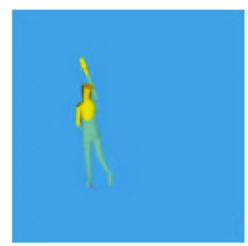

$t=12.48 \mathrm{~s}$

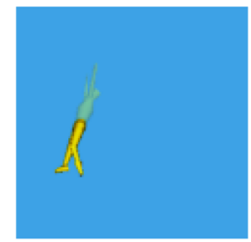

$t=14.58 \mathrm{~s}$

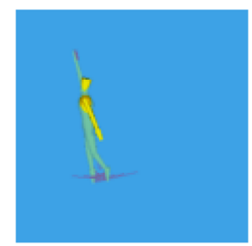

$t=12.78 \mathrm{~s}$

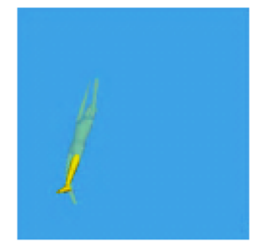

$t=14.88 \mathrm{~s}$

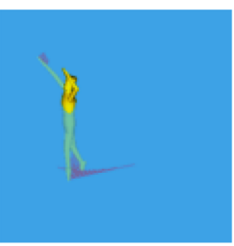

$t=13.08 \mathrm{~s}$



$t=15.18 \mathrm{~s}$

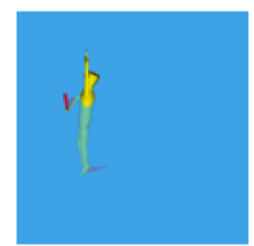

$t=13.68 \mathrm{~s}$

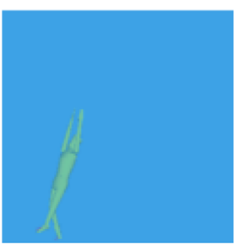

$t=15.48 \mathrm{~s}$

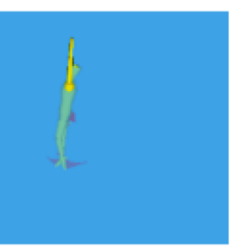

$t=13.98 \mathrm{~s}$



$t=15.78 \mathrm{~s}$



$t=14.28 \mathrm{~s}$

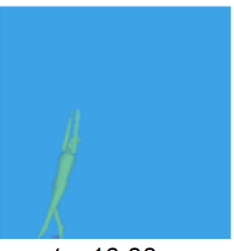

$t=16.08 \mathrm{~s}$

Fig. 23 Animation images of the simulated swimming movement (with feedback, $t=12.48 \sim 16.08 \mathrm{~s}$ ).

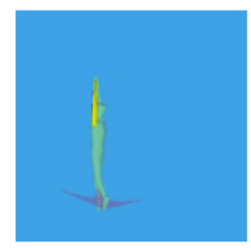

$t=18.70 \mathrm{~s}$

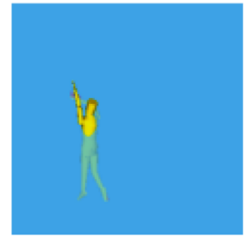

$t=20.50 \mathrm{~s}$

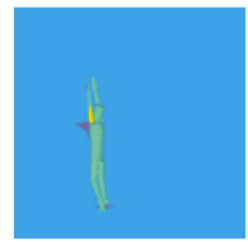

$t=19.00 \mathrm{~s}$

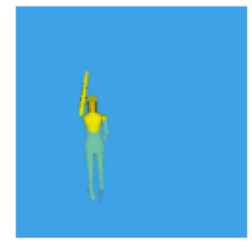

$t=20.80 \mathrm{~s}$

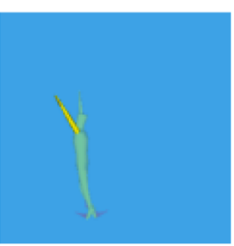

$t=19.30 \mathrm{~s}$

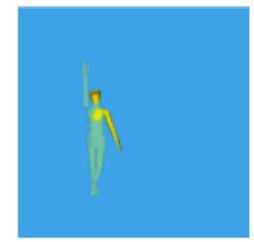

$t=21.10 \mathrm{~s}$

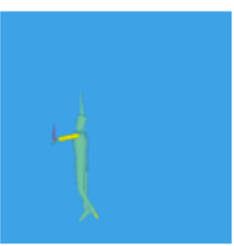

$t=19.60 \mathrm{~s}$

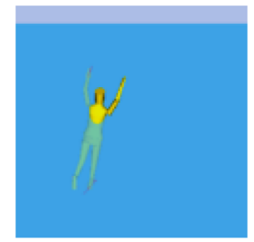

$t=21.40 \mathrm{~s}$

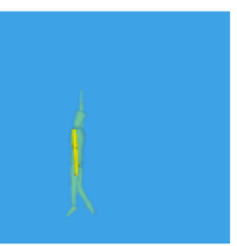

$t=19.90 \mathrm{~s}$

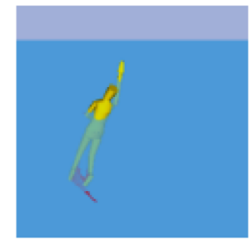

$t=21.70 \mathrm{~s}$

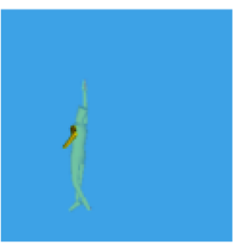

$t=20.20 \mathrm{~s}$

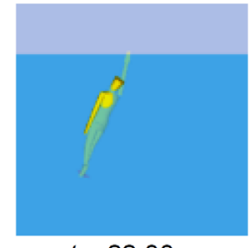

$t=22.00 \mathrm{~s}$

Fig. 24 Animation images of the simulated swimming movement (with feedback, $t=18.70 \sim 22.00 \mathrm{~s}$ ).

movement for $t=12.48 \sim 16.08 \mathrm{~s}$ and $t=18.70 \sim 22.00 \mathrm{~s}$ are shown in Figs. 23 and 24, respectively. As shown in Fig. 23 , the left arm stroke just after the disturbance was not performed. The right arm stroke was also not performed just after $t=14.28 \mathrm{~s}$. From $14.58 \mathrm{~s}$, the swimmer did not perform an arm stroke at all and "waited" for the recovery of the roll angle performing the flutter kick only. After this waiting behavior, the swimmer again started the arm stroke, as shown in Fig. 24. For $t=22 \sim 45 \mathrm{~s}$, some behaviors of delaying or not performing the arm stroke could be seen in Fig. 22 (if the output signals from the neural oscillator did not exceed 10, the arm stroke was not performed). Finally, the movement returns to the stable one completely after $45 \mathrm{~s}$. Therefore, it can be concluded that the roll angle feedback proposed in the present study was effective. Indeed, it is a remarkable advantage of the CPG network that such complex controlling behavior autonomously emerges only by constructing a simple feedback algorithm as proposed in the present study.

\section{Conclusions}

In the present study, a CPG network to generate the swimming motion of the crawl stroke was proposed. First, the CPG network for the legs performing the flutter kick was constructed by connecting the neural oscillators for the leg joints. The CPG network both for the arms and legs were constructed next, in which the neural oscillator for the arms output the trigger signal to start the prescribed stroke motion. Finally, the roll angle of the swimmer was fed back into the CPG network in order to restore the balance in the roll direction. The obtained findings are summarized as follows:

1. The flutter kick motion was successfully generated by the proposed CPG network, which had cyclic inhibitions in 
order to realize the phase lags. The propulsion by the generated flutter kick motion was confirmed by the simulation of the swimming movement.

2. The crawl stroke both by the arms and legs was successfully generated by the proposed CPG network. By changing the intrinsic cycle of the neural oscillators for the legs, both six- and two-beat crawls could be realized. The propulsion by the generated swimming motion was confirmed by the simulation of the swimming movement both for the six- and two-beat crawls.

3. A stable region with respect to the relationship between the intrinsic cycles of the neural oscillators for the arms and legs certainly existed for the six-beat crawl, although the intrinsic cycles of the arms were three times longer than those of the legs in this case.

4. Restoring the balance in the roll direction was successfully realized by the proposed feedback algorithm. The resultant motion showed a complicated behavior, such as skipping strokes.

In the present study, the output signals from the neural oscillators were directly transformed into joint angles for the legs, and were used as trigger signals of starting strokes for the arms, respectively. Indeed, these usages were not accurate from the viewpoint of neurophysiology, and were not consistent with other previous studies as well. For example, in several locomotive simulation analyses using CPG network (Taga, 1994; Hase and Yamazaki, 1999), the output from CPG network was transformed into joint moment or muscle force. Therefore, those simplifications in the present study are the limitation of the present study. The development of more neurophysiologically accurate model is an important future task.

\section{References}

Bay, J. S., and Hemami, H., Modeling of a neural pattern generators with nonlinear oscillators, Biomedical Engineering, Vol.34 (1987), pp.297-306.

Chung, C. and Nakashima, M., Development of a swimming humanoid robot for research of human swimming, Journal of Aero Aqua Bio-mechanisms, Vol.3 (2013a), pp.109-117.

Chung, C. and Nakashima, M., Free swimming of the swimming humanoid robot for the crawl stroke, Journal of Aero Aqua Biomechanisms, Vol.3 (2013b), pp.118-126.

Dietz V., Müller, R, and Colombo, G., Locomotor activity in spinal man: significance of afferent input from joint and load receptors, Brain, Vol.125 (2002), pp.2626-2634.

Dimitrievic, M. R., Gerasimenko, Y., and Pinter, M. M., Evidence for a spinal central pattern generator in humans, Annals of the New York Academy of Sciences, Vol.860 (1998), pp.360-376.

Endo, G., Nakanishi, J., Morimoto, J. and Cheng, G., Experimental studies of a neural oscillator for biped locomotion with QRIO, Proceedings of the 2005 IEEE International Conference on Robotics and Automation (2005), pp.596602.

Grillner S., Neurobiological bases of rhythmic motor acts in vertebrates, Science, Vol.228 (1985), pp.143-149.

Hase, K., and Yamazaki, N., Computational evolution of human bipedal walking by a neuro-musculo-skeletal model, Artificial Life and Robotics, Vol.3 (1999), pp.133-138.

Ijspeert, A. J., Crespi, A., Ryczko, D., and Cabelguen, J. M., From swimming to walking with a salamander robot driven by a spinal cord model, Science, Vol.315 (2007), pp.1416-1420.

Kotosaka, S., and Schaal, S., Synchronized robot drumming by neural oscillator, Journal of the Robotics Society of Japan, Vol.19 (2001), pp.116-123.

Matsuoka, K., Sustained oscillators generated by mutually inhibiting neurons with adaptation, Biological Cybernetics, Vol.52 (1985), pp.367-376.

Matsuoka, K., Mechanical of frequency and pattern control in the neural rhythm generators, Biological Cybernetics, Vol.56 (1987), pp.345-353.

Miura, N., Ikemoto, Y., Gonzalez, J., Inoue, J. and Yu, W., Analyzing compensation strategy in impaired walking using a humanoid robot, Transaction on Control and Mechanical System, Vol.1 (2012), pp.20-25.

Miyakoshi, S., Taga, G., Kuniyoshi, Y., and Nagakubo, A., Three dimensional bipedal stepping motion using neural oscillators - towards humanoid motion in the real world, Journal of the Robotics Society of Japan, Vol.18 (2000), pp.87-93. 
Nakashima, M., Satou, K., and Miura, Y., Development of swimming human simulation model considering rigid body dynamics and unsteady fluid force for whole body, Journal of Fluid Science and Technology, Vol.2 (2007), pp.5667.

Nakashima, M., Mechanical study of standard six beat front crawl swimming by using swimming human simulation model, Journal of Fluid Science and Technology, Vol.2 (2007), pp.290-301.

Nakashima, M., Modeling and Simulation of Human Swimming, Journal of Aero Aqua Bio-mechanisms, Vol.1 (2010), pp.11-17.

Shik, M. L., Severin, F. V., and Orlovsky, G. N., Control of walking by means of electrical stimulation of the mid-brain, Biophysics, Vol.11 (1966), pp.756-765.

Taga, G., Yamaguchi, Y., and Shimizu, H., Self-organized control of bipedal locomotion by neural oscillators in unpredictable environment, Biological Cybernetics, Vol.65 (1991), pp.147-159.

Taga, G., Emergence of bipedal locomotion through entrainment among the neuro-musculo-skeletal system and the environment, Physica D, Vol.75 (1994), pp.190-208.

Wannier, T., Bastiaanse, C., Colombo, G. and Dietz, V., Arm to leg coordination in humans during walking, creeping and swimming activities, Experimental Brain Research, Vol.141 (2001), pp.375-379.

Williamson, M. M., Neural control of rhythmic arm movements, Neural Networks, Vol.11 (1998), pp.1379-1394.

Wilson, H. R. and Cowan, J. D., Excitatory and inhibitory interactions in localized populations of model neurons, Biophysical Journal, Vol.12 (1972), pp.1-24.

Yang, W., Chong, N. K. and You, B. J., Biologically Inspired robot arm control using neural oscillators, Robotics 2010: Current and Future Challenges (2010), pp.133-148.

Zehr, E. P., Collins, D. F., Frigon, A., and Hoogenboom, N., Neural control of rhythmic human arm movement: phase dependence and task modulation of hoffmann reflexes in forearm muscles, Journal of Neurophysiology, Vol.89 (2003), pp.12-21.

Zehr, E. P., Carroll, T. J., Chua, R., Collins, D. F., Frigon, A., Haridas, C., Hundza, S. R., and Thompson, A. K., Possible contributions of CPG activity to the control of rhythmic human arm movement, Canadian Journal of Physiology and Pharmacology, Vol.82 (2004), pp.556-568.

Zehr, E. P., and Duysens, J., Regulation of arm and leg movement during human locomotion, The Neuroscietist, Vol.10 (2004), pp.347-361. 